\title{
Chaotic dynamics in a two-dimensional optical lattice
}

\author{
Eric Horsley, Stewart Koppell, and L. E. Reichl \\ Center for Complex Quantum Systems and Department of Physics, The University of Texas at Austin, Austin, Texas 78712, USA
}

(Received 28 October 2013; published 21 January 2014)

\begin{abstract}
The classical nonlinear dynamics of a dilute gas of rubidium atoms in an optical lattice is studied for a range of polarizations of the laser beams forming the lattice. The dynamics ranges from integrable to chaotic, and mechanisms leading to the onset of chaos in the lattice are described.
\end{abstract}

DOI: 10.1103/PhysRevE.89.012917

PACS number(s): 05.45.Ac, 42.50.Wk, 05.45.Gg

\section{INTRODUCTION}

Optical lattices are formed by superposing multiple pairs of counterpropagating lasers. When atoms are loaded into an optical lattice, the system can be used to explore quantumclassical correspondence or the dynamics of condensed matter systems [1,2].

We begin, in Sec. II, with a description of the Hamiltonian and the dynamics of atoms confined to the optical lattice. In Sec. III, we show that one major source of chaos is a bifurcation that occurs at relatively low energy. In Sec. IV, we show that a second, more dominant, source of chaos is due to the saddle points that occur throughout the lattice. Finally, in Sec. V we make some concluding remarks.

\section{OPTICAL LATTICE HAMILTONIAN}

Optical lattices have been realized in the laboratory by several experimental groups [3-5]. In the Greiner experiment [4], laser radiation (spot size of $75 \mu \mathrm{m}$, wavelength $\lambda \approx 840 \mathrm{~nm}$ ) was focused on a rubidium condensate. The Hamiltonian for the rubidium atoms, in dimensionless units, is

$$
\begin{aligned}
H= & p_{x}^{2}+p_{y}^{2}+U\left[\cos ^{2}(x)+\cos ^{2}(y)\right. \\
& +2 \alpha \cos (x) \cos (y)]=E,
\end{aligned}
$$

where $\left(p_{x}, p_{y}\right)$ and $(x, y)$ are the $x$ and $y$ components of momentum and displacement, respectively, $E$ is the total energy, $U$ is the depth of the potential (proportional to laser intensity), and $\alpha=\hat{\epsilon}_{1} \hat{\epsilon}_{2} \cos (\phi)$. The strength of the coupling $\alpha$ is determined by $\hat{\epsilon}_{1}$ and $\hat{\epsilon}_{2}$ (the polarization directions of the two laser beams) and $\phi$, the relative phase of the laser beams.

The Hamiltonian in Eq. (1) accounts for the atom-radiation interaction but neglects interactions between atoms. The relation between the dimensionless variables in Eq. (1) and dimensioned variables $\left(H^{\prime}, p_{x}^{\prime}, p_{y}^{\prime}, x^{\prime}, y^{\prime}, U^{\prime}, E^{\prime}\right)$ is $H^{\prime}=H E_{L}$, $p_{x}^{\prime}=\hbar k_{L} p_{x}, p_{y}^{\prime}=\hbar k_{L} p_{y}, x=k_{L} x^{\prime}, y=k_{L} y^{\prime}, U^{\prime}=U E_{L}$, and $E^{\prime}=E E_{L}$, where $\hbar$ is Planck's constant, $k_{L}=\frac{2 \pi}{\lambda}$ is the wave vector of the radiation, $E_{L}=\frac{\hbar^{2} k_{L}^{2}}{2 m_{R b}}=2.156 \times 10^{-30} \mathrm{~J}$ is the recoil energy of rubidium, and $m_{R b} \stackrel{2 m_{R b}}{=} 86.909 \mathrm{u}$ is the mass of ${ }^{87} \mathrm{Rb}$. We analyze the dynamics for the interval $0 \leqslant \alpha \leqslant 1.0$ and with $U=20$, a value attainable in current experiments [6].

In Fig. 1, we show contour plots of the potential energy surface for one unit cell of the lattice for $\alpha=$ $0.1, \alpha=0.5$, and $\alpha=1.0$ (four unit cells for $\alpha=0$ ). The lattice has discrete symmetries. The Hamiltonian Eq. (1) is invariant under reflections $(x \rightarrow-x, y \rightarrow-y)$, under rotations $(x \rightarrow \pm y, y \rightarrow \pm x)$, and under translations $(x \rightarrow x \pm 2 \pi, y \rightarrow y \pm 2 \pi)$ and $(x, y) \rightarrow(x \pm \pi, y \pm \pi)$. The unit cell can therefore be divided into 16 identical fundamental triangles (FTs), each containing full information about the classical dynamics of the lattice. Two of the FTs are shown in the top right quadrant of Fig. 1(c). For $0<\alpha<1$, each FT has one potential energy absolute maximum, one absolute minimum, one potential energy saddle point, and a line of local potential energy minima (a "trench") running from the saddle point to the potential energy minimum. The equation locating the trenches is $y_{T}(x)=\cos ^{-1}[ \pm \alpha \cos (x)]$ (the sign varies with saddle point).

Figure 1 shows a unit cell (for $\alpha>0$ ). When $\alpha=0$ there are nine potential energy maxima. As $\alpha$ increases, five maxima grow in height, and four shrink and disappear at $\alpha=1.0$. There are four potential energy absolute minima unchanged for all values of $\alpha$, and there are 12 saddle points. As $\alpha$ increases, the saddles move toward the four low maxima and finally, at $\alpha=$ 1.0, they merge with low maxima and disappear. At $\alpha=1.0$, there are five potential energy maxima $V_{\max }(1.0)=4 U$ and four lines of zero potential energy between $(x, y)=(0, \pm \pi)$ and $(x, y)=( \pm \pi, 0)$.

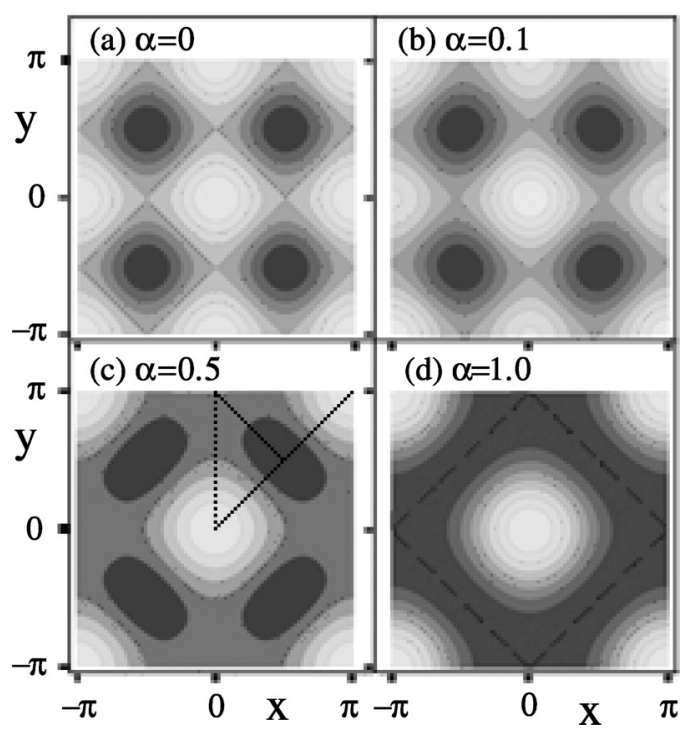

FIG. 1. Contour plots of a unit cell of the potential energy $V(x, y)$ for (a) $\alpha=0$ (four unit cells), (b) $\alpha=0.1$, (c) $\alpha=0.5$, and (d) $\alpha=1.0$. In Fig. 1(c), 2 of the 16 fundamental triangles in the unit cell are indicated by dashed lines. All quantities are in dimensionless units. 


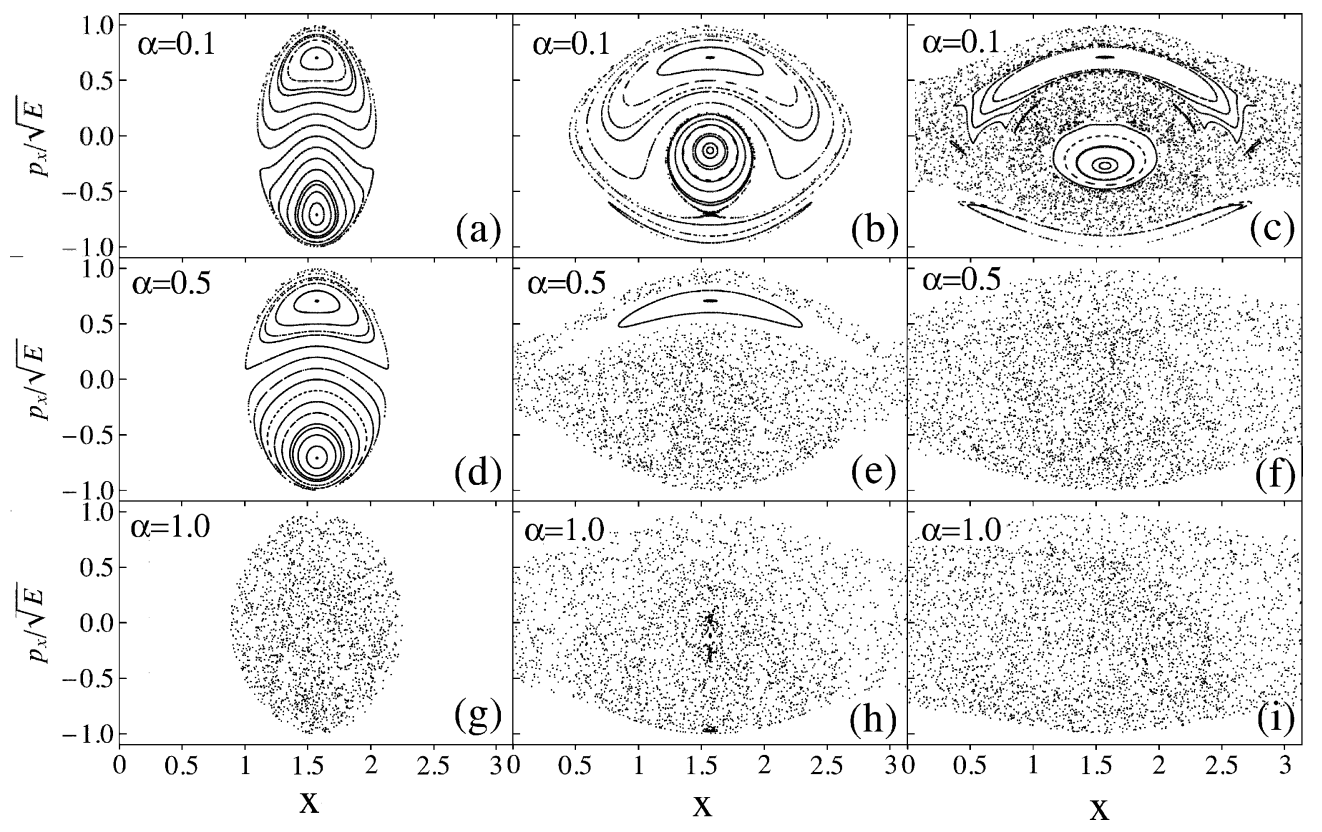

FIG. 2. PSS of $p_{x} / \sqrt{E}$ vs $x$ plotted each time $y=\pi / 2$ for $p_{y}>0$. (a) $\alpha=0.1, E=\frac{1}{10} V_{\max }$. (b) $\alpha=0.1, E=\frac{3}{8} V_{\max }$. (c) $\alpha=0.1$, $E=\frac{5}{8} V_{\max }$. (d) $\alpha=0.5, E=\frac{1}{10} V_{\max }$. (e) $\alpha=0.5, E=\frac{3}{8} V_{\max }$. (f) $\alpha=0.5, E=\frac{5}{8} V_{\max }$. (g) $\alpha=1.0, E=\frac{1}{10} V_{\max }$. (h) $\alpha=1.0, E=\frac{3}{8} V_{\max }$. (i) $\alpha=1.0, E=\frac{5}{8} V_{\max }$. All quantities are in dimensionless units.

In Fig. 2, we show the Poincaré surface of section (PSS) $\frac{p_{x}}{\sqrt{E}}$ versus $x$, plotted each time $y=\pi / 2$ for $p_{y}>0$. Figure 2 contains nine plots: three plots with $\alpha=0.1, \alpha=0.5$, and $\alpha=$ 1.0 for each of the energies $E=\frac{1}{10} V_{\max }(\alpha), E=\frac{3}{8} V_{\max }(\alpha)$, and $E=\frac{5}{8} V_{\max }(\alpha)$, where $V_{\max }(\alpha)$ is the potential energy maximum for a given value of $\alpha\left[V_{\max }(0.1)=44, V_{\max }(0.5)=\right.$ 60 , and $\left.V_{\max }(1.0)=80\right]$.

For $\alpha=0.1$ and energies $E<\frac{5}{8} V_{\max }(0.1)$ [Figs. 2(a) and 2(b)], the phase space dynamics is dominated by Kolmogorov-Arnold-Moser (KAM) tori. At about $E=6.86$, there is a bifurcation of one of the stable periodic orbits. At the bifurcation, an unstable periodic orbit emerges and is the primary source of chaos at low energies.

When $E$ becomes greater than the saddle point energy, the unstable fixed point at the saddle points provide a second source of chaos. The broad chaotic sea in Fig. 2(c) is generated by the bifurcation and the saddle point. For $\alpha=0.5$, the bifurcation occurs at higher energy, and the saddle point has lower energy. Figures 2(d)-2(f) show the evolution of the phase space dynamics for $\alpha=0.5$ for increasing energies. In Fig. 2(d) $(E=6.0)$, the system is well below the bifurcation energy. In Fig. 2(e) $(E=22.5)$, both the bifurcation and the saddle point contribute to the chaos. In Fig. 2(f) $(E=37.5)$, the dynamics is chaotic. For $\alpha=1.0$, the PSSs in Figs. 2(g)2(i) show that the phase space dynamics is chaotic for all energies below $V_{\max }(1.0)$.

We can estimate the number of quantum states in a unit cell of the lattice at a given energy $E$ from the phase space volume occupied by the classical orbits. For a given energy $E$, we have $\frac{1}{2 \pi} \oint p_{x} d x \approx n_{x}$ and $\frac{1}{2 \pi} \oint p_{y} d y \approx n_{y}$, so the number of quantum states is $n_{x} n_{y}$. In Fig. 2(f), the area of the chaotic region is approximately $\left(\Delta p_{x}\right)(\Delta x) \sqrt{E} \approx 33$, and the number of quantum states is $n_{x}=\frac{33}{2 \pi} \approx 5.3$.

\section{WALKER-FORD BIFURCATION}

For small $\alpha$, we can use Walker and Ford analysis [7] to describe the bifurcation. When $\alpha=0$, the dynamics consists of two uncoupled pendulums with total Hamiltonian $H=H_{x}+$ $H_{y}=E$, where $H_{x}=p_{x}^{2}+U \cos ^{2}(x)=E_{x}$ and $H_{y}=p_{y}^{2}+$ $U \cos ^{2}(y)=E_{y}$. For energy $E<20$, the motion is librational, and the action variable, for dynamics $H_{x}=E_{x}$, is

$$
J_{x}=\frac{1}{2 \pi} \oint d x p_{x}=\frac{2}{\pi} \sqrt{U}\left[\mathrm{E}\left(\kappa_{x}\right)-\left(1-\kappa_{x}^{2}\right) \mathrm{K}\left(\kappa_{x}\right)\right],
$$

where the modulus $\kappa_{x}^{2}=E_{x} / U$ and $\mathrm{K}(\kappa)$ and $\mathrm{E}(\kappa)$ are complete elliptic integrals of the first and second kind, respectively. The canonical transformation $\left(p_{x}, x\right) \rightarrow\left(J_{x}, \theta_{x}\right)$ [8] is given by $x=\frac{\pi}{2}+\sin ^{-1}\left[\kappa_{x} \operatorname{sn}\left(f_{x}, \kappa_{x}\right)\right]$ and $p_{x}=\sqrt{U} \kappa_{x} \operatorname{cn}\left(f_{x}, \kappa_{x}\right)$, where $\operatorname{sn}\left(f_{x}, \kappa_{x}\right)$ and $\operatorname{cn}\left(f_{x}, \kappa_{x}\right)$ are Jacobi sn and cn functions, respectively, and $f_{x}=\frac{2}{\pi} \mathrm{K}\left(\kappa_{x}\right) \theta_{x}$. An identical analysis applies to $H_{y}$.

In terms of the action-angle variables, the Hamiltonian in Eq. (1) can be written

$$
\begin{aligned}
H= & E_{x}+E_{y}+2 U \kappa_{x} \kappa_{y} \alpha \operatorname{sn}\left(f_{x}, \kappa_{x}\right) \operatorname{sn}\left(f_{y}, \kappa_{y}\right) \\
= & E_{x}+E_{y}+\alpha U \frac{\pi^{2}}{\mathrm{~K}\left(\kappa_{x}\right) \mathrm{K}\left(\kappa_{y}\right)} \sum_{n_{x}=0}^{\infty} \sum_{n_{y}=0}^{\infty} \sum_{\beta= \pm 1} C_{n_{x}}\left(\kappa_{x}\right) \\
& \times C_{n_{x}}\left(\kappa_{x}\right) \cos \left[\left(2 n_{x}+1\right) \theta_{x}+\beta\left(2 n_{y}+1\right) \theta_{y}\right],
\end{aligned}
$$

where $\quad C_{n_{x}}\left(\kappa_{x}\right)=\operatorname{csch}\left[\left(2 n_{x}+1\right) \frac{\pi}{2} \frac{\mathrm{K}^{\prime}\left(\kappa_{x}\right)}{\mathrm{K}\left(\kappa_{x}\right)}\right] \quad$ and $\quad \mathrm{K}^{\prime}\left(\kappa_{x}\right)=$ $\mathrm{K}\left(\sqrt{1-\kappa_{x}^{2}}\right)$, with $C_{n_{y}}\left(\kappa_{y}\right)$ and $\mathrm{K}^{\prime}\left(\kappa_{y}\right)$ defined in a similar manner. Each term in the summation in Eq. (3) induces a "primary resonance" in the phase space. The resonances accumulate at the separatrices of the pendulum and generate a stochastic layer [8]. The largest resonance $\left[\left(n_{x}, n_{y}\right)=(0,0)\right]$ lies farthest from the separatrices. 

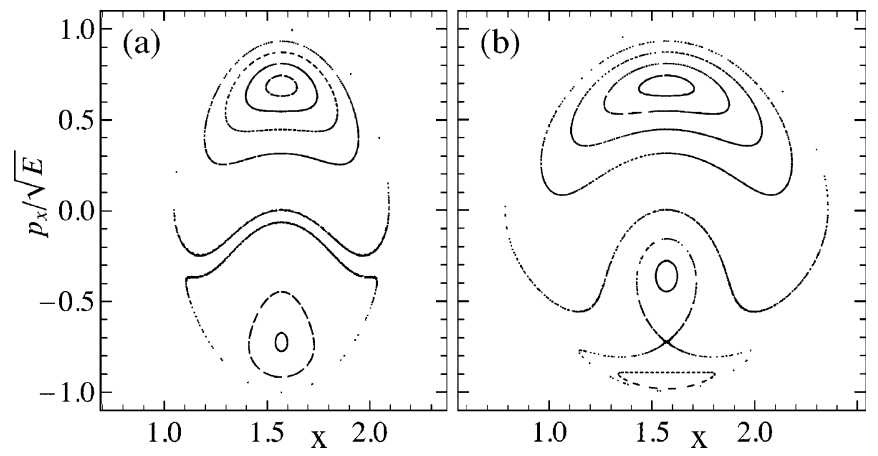

FIG. 3. Surfaces of section for the Walker-Ford Hamiltonian for $\alpha=0.1$ and transformed to Cartesian coordinates. The PSS shows $\left(\frac{p_{x}}{\sqrt{E}}, x\right)$ (for $y=\frac{\pi}{2}$ and $p_{y}>0$ ). (a) $E=5.0$. (b) $E=10$. All quantities are in dimensionless units.

We now examine the effect of the largest primary resonance on the phase space structure. Isolate the term in Eq. (3) with $\left(n_{x}, n_{y}\right)=(0,0)$ and $\beta=-1$ and write

$$
\begin{aligned}
H_{0,0}^{(-)}= & E_{x}+E_{y}+\alpha U \frac{\pi^{2}}{\mathrm{~K}\left(\kappa_{x}\right) \mathrm{K}\left(\kappa_{y}\right)} C_{0}\left(\kappa_{x}\right) C_{0}\left(\kappa_{y}\right) \\
& \times \cos \left[\theta_{x}-\theta_{y}\right]=E .
\end{aligned}
$$

Given $H_{0,0}^{(-)}$, we solve Hamilton's equations for $\left(J_{x}, J_{y}, \theta_{x}, \theta_{y}\right)$ and transform the solutions to Cartesian coordinates. In Fig. 3, we plot a PSS of $\left(\frac{p_{x}}{\sqrt{E}}, x\right)$ (for $y=\frac{\pi}{2}$ and $p_{y}>0$ ) for $\alpha=0.1$. Below $E=6.86$, the phase space shows no unstable periodic orbits (UPO), while for $E>6.86$, the primary resonance [Eq. (4)] emerges and is the source of the bifurcation [compare Fig. 2(a) with Fig. 3(a) and Fig. 2(b) with Fig. 3(b)].

\section{SADDLE POINT CHAOS}

Another source of chaos is the saddle points. Consider the four saddle points at $\left(x_{s p}, y_{s p}\right)=\left(0, \cos ^{-1}(-\alpha)\right)$, $\left(\pi, \cos ^{-1}(+\alpha)\right),\left(\cos ^{-1}(-\alpha), 0\right)$, and $\left(\cos ^{-1}(+\alpha), \pi\right)$. The linearized equations of motion in the neighborhood of $\left(0, \cos ^{-1}(-\alpha)\right)$, exhibits harmonic motion in the $y$ direction
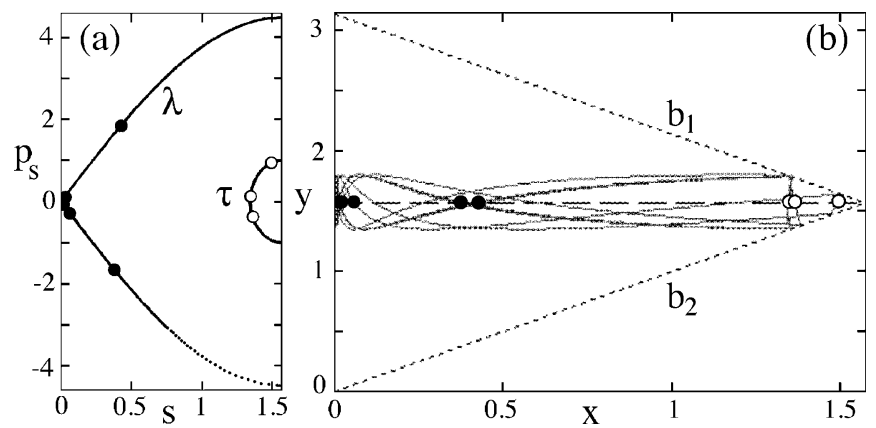

FIG. 4. PSS of stable and unstable manifolds for $\alpha=0$ and energy $E=E_{s p}(\alpha)+1.0$, along the trench with saddle $\left(x_{s p}, y_{s p}\right)=$ $\left(0, \frac{\pi}{2}\right)$. (a) The separatrix of the UPO at $\left(x_{s p}, y_{s p}\right)=\left(0, \frac{\pi}{2}\right)$. (b) The FT in configuration space and the configuration space orbit of points marked with open and solid circles in (a). Points $\tau$ come from the separatrix between $\left(x_{s p}, y_{s p}\right)=\left(\frac{\pi}{2}, 0\right)$ and $\left(\frac{\pi}{2}, \pi\right)$. Points $\lambda$ come from the separatrix between $\left(x_{s p}, y_{s p}\right)=\left(\frac{\pi}{2}, 0\right)$ and $\left(\frac{\pi}{2}, \pi\right)$. All quantities are in dimensionless units. and unstable motion in the $x$ direction with deviations from the saddle fixed point given by $\Delta x=A e^{-2 \sqrt{U\left(1-\alpha^{2}\right) t}}$ and $\Delta p_{x}=-2 A \sqrt{U\left(1-\alpha^{2}\right)} e^{-2 \sqrt{U\left(1-\alpha^{2}\right) t}}$. The unstable (stable) manifold thus departs forward (backward) in time from the saddle point $\left(x_{s p}, y_{s p}\right)=\left(0, \cos ^{-1}(-\alpha)\right)$ in the $x-p_{x}$ plane.

For $\alpha=0$, the unstable (stable) manifold of $\left(x_{s p}, y_{s p}\right)=$ $\left(0, \frac{\pi}{2}\right)$ forms a separatrix on the $y=\frac{\pi}{2}$ PSS which connects to the saddle point at $\left(x_{s p}, y_{s p}\right)=\left(\pi, \frac{\pi}{2}\right)$. Stable and unstable manifolds also connect saddle points $\left(x_{s p}, y_{s p}\right)=\left(\frac{\pi}{2}, 0\right)$ and $\left(x_{s p}, y_{s p}\right)=\left(\frac{\pi}{2}, \pi\right)$. For all $\alpha$, these manifolds are unique curves with the same energy as the saddle unstable fixed point (UFP). When $0<\alpha<1$, the separatrices from neighboring saddle points form oscillatory area preserving curves (tendrils) that transversally intersect the separatrices of neighboring saddles and contain complete information about the chaotic region.

For $\alpha \neq 0$, there is no PSS of $\left(\frac{p_{x}}{\sqrt{E}}, x\right)$ for fixed $y$ that contains the complete invariant manifold associated with the saddles. However, we can construct a PSS along the trench (using Birkhoff coordinates) that does contain the invariant

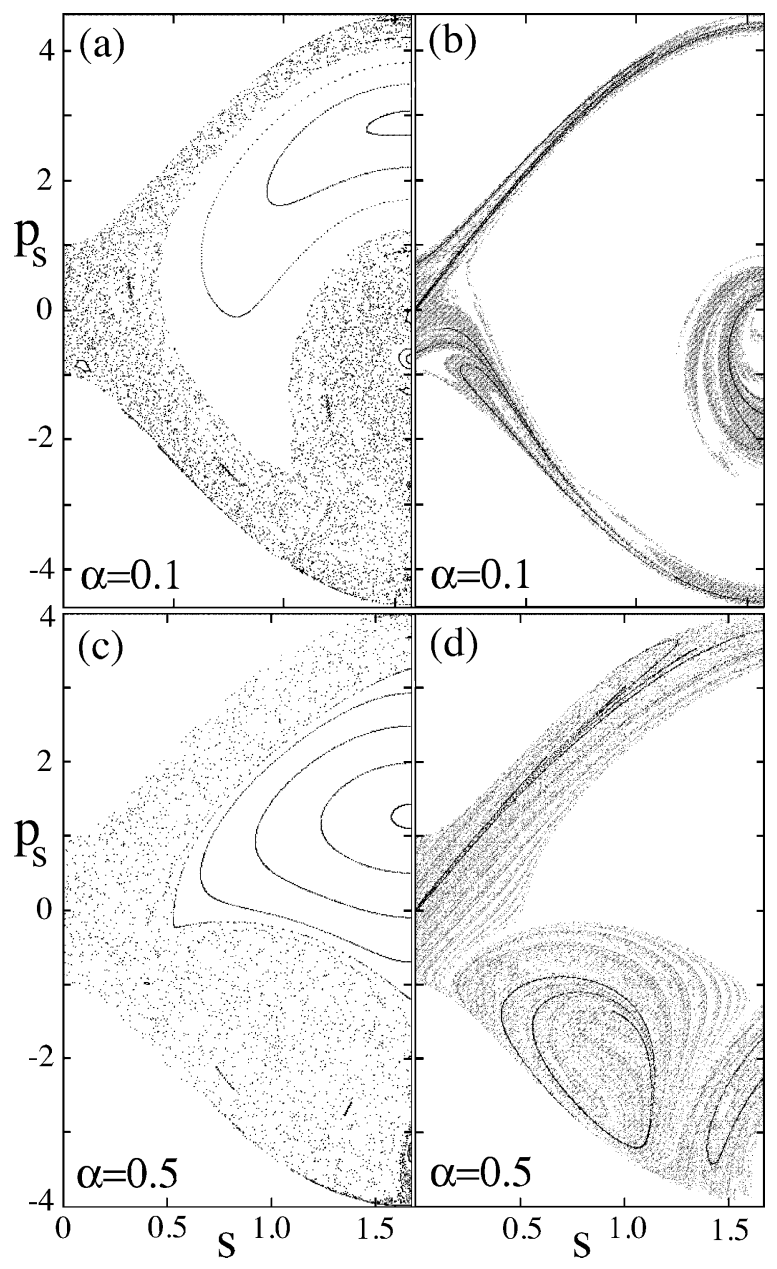

FIG. 5. PSSs of Birkhoff coordinates $\left(p_{s}, s\right)$ along the "trench" between $\left(x_{s p}, y_{s p}\right)=\left(0, \frac{\pi}{2}\right)$ and $\left(x_{\min }, y_{\min }\right)=\left(\frac{\pi}{2}, \frac{\pi}{2}\right)$ for $E=$ $E_{s p}(\alpha)+1.0$. (a) Chaotic region for $\alpha=0.1$. (b) Stable and unstable manifolds for $\alpha=0.1$. (c) Chaotic region for $\alpha=0.5$. (d) Stable and unstable manifolds for $\alpha=0.5$. All quantities are in dimensionless units. 
manifolds. The PSS using Birkhoff coordinates maps the component of momentum, $p_{s}$, tangent to the trench versus the position, $s$, along the trench as a trajectory passes through the trench at position $s$. A PSS using Birkhoff coordinates is area preserving.

A PSS along a "trench" has unusual properties that we illustrate for $\alpha=0$, when the trench coincides with $y=\frac{\pi}{2}$. We assume the FT has hard wall boundary conditions. In the PSS in Fig. 4(a), we plot the stable and unstable manifolds for the saddle fixed point at $\left(x_{s p}, y_{s p}\right)=\left(0, \frac{\pi}{2}\right)$ for $\alpha=0$. The saddle point energy is $E_{s p}(\alpha)$, but the unstable fixed point at the saddle also exists at higher energy [the "excess energy" $\left.\Delta E(\alpha)=E-E_{s p}(\alpha)\right]$. For $\alpha=0$, the stable and unstable manifolds for the saddle fixed point at $\left(x_{s p}, y_{s p}\right)=\left(0, \frac{\pi}{2}\right)$ form a separatrix [see Fig. 4(a) for $\Delta E(0)=1.0$ ]. The unstable manifold is computed from a line of initial conditions along the positive eigencurve of the saddle fixed point. The evolution of a single initial condition is shown in configuration space in Fig. 4(b). The dashed curves $\left(b_{1}\right.$ and $\left.b_{2}\right)$ are the boundaries of the FT. The solid gray line is the trajectory, and the dots are points where it crosses $y=\frac{\pi}{2}$ and is plotted in the PSS. The FT PSS has contributions from unstable manifolds of all four saddle UPOs. When the trajectory hits the wall of the FT, it reflects and crosses the line $y=\frac{\pi}{2}$ vertically (the open dots) and reflects again before it continues its path back to the saddle. The vertical segments correspond to sections of the stable and unstable manifolds of the saddles at $\left(x_{s p}, y_{s p}\right)=\left(\frac{\pi}{2}, 0\right)$ and $\left(\frac{\pi}{2}, \pi\right)$ (marked $\tau$ ). The black dots mark sections of the stable and unstable manifolds of the saddles at $\left(x_{s p}, y_{s p}\right)=\left(0, \frac{\pi}{2}\right)$ and $\left(\pi, \frac{\pi}{2}\right)(\operatorname{marked} \lambda)$.

In Fig. 5(a), we show the phase space structure of the FT PSS for an excess energy of $\Delta E(\alpha)=1.0$ and coupling $\alpha=$ 0.1. In Fig. 5(b), we show the stable and unstable manifolds associated with the four saddle UFPs that contribute to this FT PSS. The first 4 mappings are marked by the dark curve, and the next 16 are shown in gray. Figure 5(c) shows the phase space structure of the FT PSS for $\Delta E(\alpha)=1.0$ and $\alpha=0.5$. The chaotic region is now almost completely dominated by the unstable manifold. In Fig. 5(d), we plot 20 iterations of a line of initial conditions. The first 4 iterations are the dark curve, and the next 16 are the gray curve.

In Fig. 6, we show the same chaotic region shown in Fig. 5(a) but for different (carefully chosen) initial conditions. There are two distinct chaotic regions, one [Fig. 6(a)] associated with the saddle point unstable manifold and the other [Fig. 6(b)] associated with the bifurcation.

When $\alpha \rightarrow 1$ [Figs. 2(g)-2(i)] the phase space dynamics becomes chaotic for $E<V_{\max }(1.0)$; however, it is not truly a

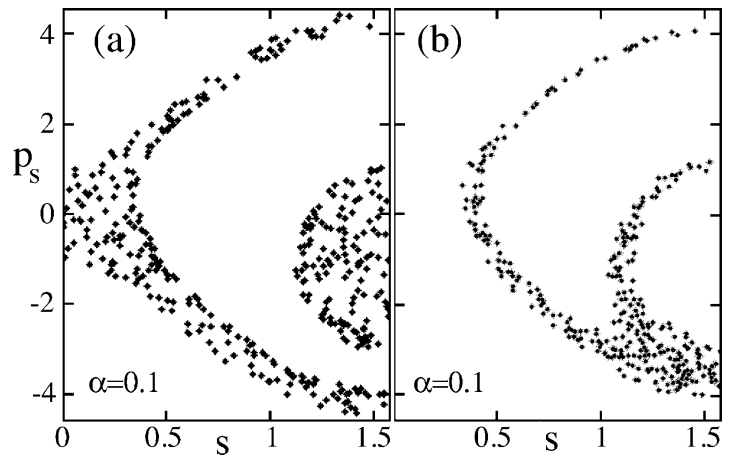

FIG. 6. Two distinct, significant chaotic regions for $E<V_{\max }$ and $\alpha=0.1$. (a) Saddle point chaos. (b) Bifurcation related chaos. All quantities are in dimensionless units.

$K$ flow (fully chaotic) and will have a mixed phase space at small scales. For $\alpha=1$, the optical lattice dynamics for $E<$ $V_{\max }(1.0)$ is locally similar to the quartic potential $V(x, y)=$ $\frac{1}{2} x^{2} y^{2}[9,10]$, whose dynamics was shown to be a $K$ flow [11], and it has some resemblance to a Lorentz gas, whose dynamics is rigorously a $K$ flow $[12,13]$. Since the quantum dynamics only "sees" classical structures that are greater than $\hbar^{2}$, atoms in the chaotic regions of the optical lattice phase space will behave as if the system is truly a $K$ flow.

\section{CONCLUDING REMARKS}

We can use the same type of analysis to study the atomic dynamics for $E>V_{\max }(\alpha)$. For $\alpha>0$, we find that a chaotic sea drives the dynamics for energies just above $V_{\max }(\alpha)$. For $\alpha=1.0$, large resonances continue to dominate the phase up to very high energies $\left[E=10 V_{\max }(\alpha)\right]$; however, chaos no longer plays a significant role at those high energies.

The optical lattice considered here contains the entire range of dynamical behaviors found in conservative systems. The fact that the optical lattices dynamics is "tunable" makes them extremely versatile systems for studying issues related to the classical-quantum correspondence for two-dimensional and three-dimensional systems and the dynamics of condensed matter systems.

\section{ACKNOWLEDGMENTS}

The authors thank the Robert A. Welch Foundation (Grant No. F-1051) for support of this work. We also thank Christof Jung for enlightening and thought-provoking discussions.
[1] I. Bloch, Nat. Phys. 1, 25 (2005).

[2] M. Lewenstein, A. Sanpera, V. Ahufinger, B. Damski, A. Sen, and U. Sen, Adv. Phys. 56, 243 (2007).

[3] A. Hemmerich, D. Schropp, and T. W. Hansch, Phys. Rev. A 44, 1910 (1991).

[4] M. Greiner, I. Bloch, O. Mandel, T. W. Hänsch, and T. Esslinger, Appl. Phys. B 73, 769 (2001); Phys. Rev. Lett. 87, 160405 (2001).
[5] I. Bloch, J. Dalibard, and S. Nascimbene, Nat. Phys. 8, 267 (2012).

[6] M. Greiner, O. Mandel, T. Esslinger, T. W. Hansch, and I. Bloch, Nature (London) 415, 39 (2002).

[7] G. H. Walker and J. Ford, Phys. Rev. 188, 416 (1969).

[8] L. E. Reichl, The Transition to Chaos, 2nd ed. (Springer, New York, 2004). 
[9] A. Carnegie and I. C. Percival, J. Phys. A 17, 801 (1984).

[10] M. P. Joy and M. Sabir, Pramana 40, 17 (1993).

[11] G. K. Savvidy, Nucl. Phys. B 246, 302 (1984).
[12] T. Geisel, A. Zacherl, and G. Radons, Z. Phys. B Conden. Matter 71, 117 (1988).

[13] P. Gaspard and F. Baras, Phys. Rev. E 51, 5332 (1995). 